\title{
Eficiência de um Treino de Leitura Crítica em Pós-Graduandos de Diferentes Áreas
}

Eficiência de um treino de leitura critical reading training efficiency in graduate students of different areas

Eficiencia de un entrenamiento de lectura crítica en post-graduados de diferentes áreas

Carmen Lúcia Hussein

Universidade de Taubaté

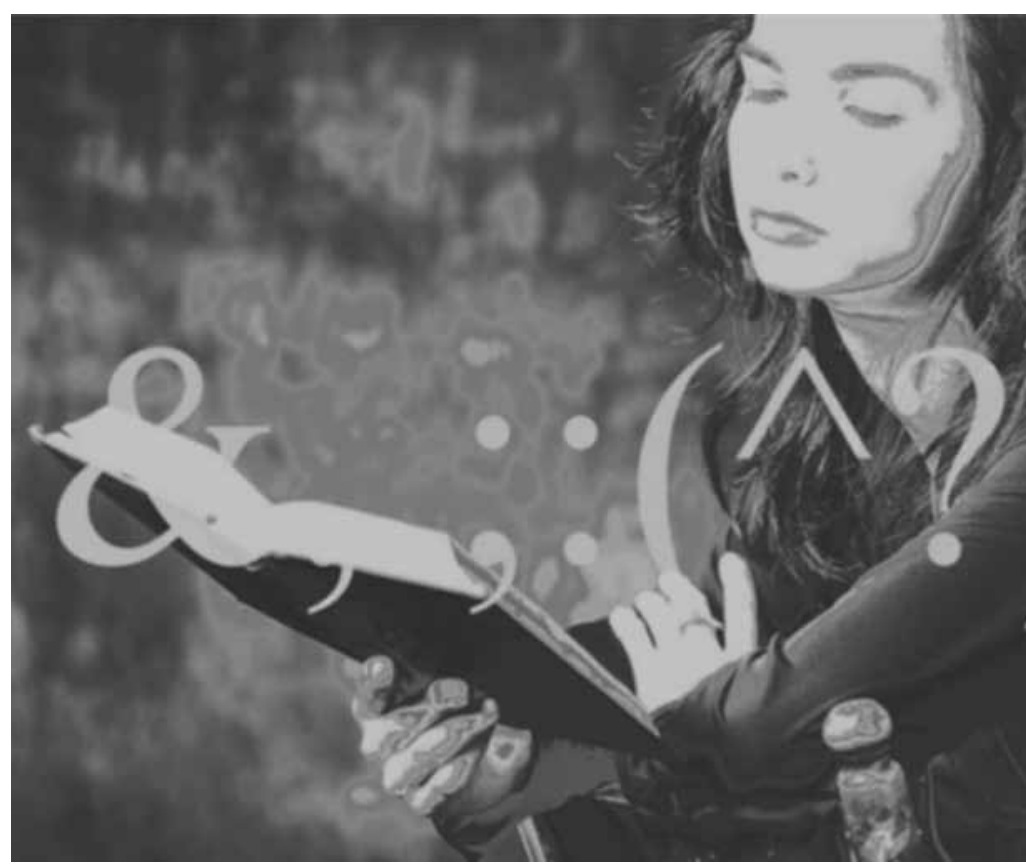


Resumo: $\mathrm{O}$ objetivo deste trabalho foi testar a eficiência de um treino de leitura crítica em pós-graduandos de diferentes áreas, e ainda o de avaliar a compreensão de textos comparando cinco grupos. O número de participantes foi de 20 alunos, oriundos de duas classes de um mesmo curso, ministrado por um único professor. Foram usados cinco grupos de alunos: G.I (humanas), G.II (Economia), G.III (Engenharia), G.IV (saúde) e G.V (Letras). Um mesmo treino de leitura crítica foi aplicado a todos os grupos. O treino foi constituído de questões, e a audiência foi fornecida pela experimentadora para fortalecer a classe de respostas críticas. Foi aplicado o Teste Cloze no pré e no pós-teste. Os dados obtidos demonstraram que não houve diferenças significantivas entre os grupos no pré e pós; também mostram a eficiência do treino oferecido para todos os grupos. Pode-se concluir que o treino foi eficiente para todas as áreas igualmente, e que, portanto, tal treino foi independente das áreas estudadas.

Palavras-chave: Aprendizagem verbal. Aprendizagem da leitura. Compreensão textual. Leitura crítica.

Abstract: In this work the efficiency of a particular training program in critical reading of graduate students in different areas of research was tested. Five groups of these graduate students were evaluated so far as comprehension of texts is concerned. The students who participated in the work belonged to two classes of the same course given by the same professor. The five groups of graduate students were classified as G.I. (Humanities), G.II (Economics), G.III (Engineering), G.IV (Health Sciences) and G.V (Literature). The same type of training in critical reading was used in all five groups. The training consisted of questions and written reforcement supplied by the examiner to enhance the critical response of the university students. The Cloze Test was applied to pre and post-test. The collected data indicated no significant differences among the groups in the pre-and post-test. The data also demonstrated the efficiency of the training given to all groups. One may conclude that the training was equally efficient to all groupss, and, consequently, such training is area-independent.

Keywords: Verbal learning. Reading learning. Textual comprehension. Critical reading.

Resumen: El objetivo de este trabajo fue probar la eficiencia de un entrenamiento de lectura crítica en postgraduados de diferentes áreas, y aún el de evaluar la comprensión de textos comparando cinco grupos. El número de participantes fue de 20 alumnos, oriundos de dos clases de un mismo curso, impartido por un único maestro. Fueron usados cinco grupos de alumnos: G.I (humanas), G.II (Economía), G.III (Ingeniería), G.IV (salud) y G.V (Letras). Un mismo entrenamiento de lectura crítica fue aplicado a todos los grupos. El entrenamiento fue constituido de cuestiones, y la audiencia fue suministrado por la experimentadora para fortalecer la clase de respuestas críticas. Fue aplicada la Prueba Cloze en el pre y en la pos-prueba. Los datos logrados demostraron que no hubo diferencias significativas entre los grupos en el pre y pos; también muestran la eficiencia del entrenamiento ofrecido para todos los grupos. Se puede concluir que el entrenamiento fue eficiente para todas las áreas igualmente, y que, por tanto, tal entrenamiento fue independiente de las áreas estudiadas.

Palabras-clave: Aprendizaje verbal. Aprendizaje de la lectura. Comprensión textual. Lectura crítica.

Estudos recentes parecem mostrar a mesma indicação de que o repertório do aluno em nível superior apresenta dificuldades para a compreensão de texto, sugerindo, portanto, que a Universidade atualmente tem recebido alunos com limitações em sua formação anterior (Ferreira \& Dias, 2002; Oliveira \& Santos, 2005, 2006; Sampaio \& Santos, 2002; Santos, 1989, 1991; Tessaro, 2004; Witter, 1997), evidenciando a necessidade de pesquisas posteriores em relação a essa questão na pós-graduação.
Em nosso meio, há uma política de democratização do ensino que facilita o ingresso na Universidade, não dando a esta a função de reorganizar o processo de leitura e o desempenho em todas as atividades cognitivas dos alunos. Assim, com o aumento do número de vagas, os alunos chegam às universidades com certa lacuna na leitura, o que é prejudicial, pois há pesquisas que mostram a relação entre competência de leitura e desempenho acadêmico (Marini, 1986). 
Witter (1999) afirma que há carência depesquisas de avaliação dos programas de leitura, de pesquisas que testem a eficiência destes, de materiais de leitura e de pesquisa de leitura na sala de aula.
Segundo Hussein (1999, 2007), a leitura crítica e a leitura criativa não têm recebido a atenção merecida, principalmente no ensino superior, embora essas habilidades sejam muito importantes na educação do indivíduo e devessem ser uma questão primordial na Universidade.

Halpern e Nummedal (1995) afirmam que o ensino superior americano demonstra que menos da metade dos estudantes que entravam nas universidades estavam aptos a usar os processos de raciocínio formal e de pensamento crítico de modo satisfatório. Sugerem, ainda, que, sem instrução explícita em pensamento crítico, somente uma pequena parcela de alunos universitários desenvolve essa habilidade.

A leitura crítica tem, nos estudos recentes, sido classificada como pensamento crítico. As habilidades de pensamento crítico descritas nos textos dessa área são semelhantes às habilidades em texto de leitura crítica (Douglas, 2000). Assim, no presente estudo, vamos considerar o pensamento crítico em face de textos e leitura crítica como sinônimos.

Vários autores reconhecem que não há uma fórmula mágica para desenvolver o pensamento crítico quanto a textos (Wade, 1995). Há uma variedade de técnicas

de leitura na sala de aula. Assim, foram encontrados alguns estudos que usaram programas com questões que requerem processos cognitivos superiores para o ensino de comportamento de leitura crítica de alunos universitários (Connor-Greene, 2005; Sainsbury, 2006).

Vários autores enfatizam que a natureza das questões possibilita um efeito sobre o desenvolvimento do pensamento na classe (Halpern \& Numendal, 1995; Wolf, Crosson, \& Resnick, 2005). Portanto, as questões formuladas pelo texto apontam os limites dentro dos quais os alunos podem operar e o nível de complexidade das operações cognitivas a serem realizadas (Pailliotet, Semali, Rodenberg, Giles, \& Macaul, 2000). Applegate, Quinn e Applegate (2002) analisaram oito inventários de leitura informal quanto a inferência de alto e baixo nível. Cerca de 91\% dos itens dos inventários foram classificados como de baixo nível ou literal, requerendo somente que o leitor se lembre das informações do texto. Itens de inferência de alto nível foram minoria.

Os autores acima afirmam que os dados de várias pesquisas sugerem que o questionamento em sala de aula é amplamente literal, em sua maioria. Assim, a ênfase é colocada quase sempre na memorização de fatos e no que os alunos devem responder nos testes. apresentadas na edição especial de Teaching of Psychology (Halpern \& Nummedal, 1995) sobre o ensino de pensamento crítico em face de textos.

Witter (1999) afirma que há carência de pesquisas de avaliação dos programas de leitura, de pesquisas que testem a eficiência destes, de materiais de leitura e de pesquisa
Usando questões, Hussein (1999) realizou um estudo que teve como objetivo testar a eficiência de procedimentos de treino em leitura crítica e leitura criativa. Foram usados 20 alunos de $1^{\circ}$ ano de Psicologia, tendo dois grupos experimentais sido submetidos ao treino de leitura crítica e de leitura criativa. Os dados obtidos demonstraram 
a eficiência dos treinos oferecidos para as habilidades crítica e criativa. Quanto à generalização, pode-se dizer que o grupo submetido ao treino de criticidade conduziu à produção de criatividade textual. Todavia, o procedimento de treino de leitura criativa não afetou o comportamento de criticidade textual. Finalmente, a variável modalidade de treino não produziu evidências de melhora nos comportamentos de compreensão e motivação de leitura.

Connor-Greene (2005) usou um método de ensino (QQTP), constituído de questões, com trechos de leitura que apresentavam controvérsias e a realização de um breve sumário para desenvolver o pensamento crítico e criativo em face de textos. Esse método teve como objetivo encorajar habilidades criativas, analíticas e solução de problemas. Ele também solicita dos alunos que interajam com os textos de leitura, articulem as idéias e despertem questões com significado. A autora conclui que esse método é efetivo para o desenvolvimento de pensamento crítico e criativo em face de textos e serve de preparação para a discussão em sala de aula. Também conclui que a discussão em sala de aula pode aumentar essas habilidades e também a de comunicação em universitários.

Hussein (2007) realizou um trabalho com o objetivo de testar a eficiência do procedimento de treino em leitura compreensiva e leitura crítica. Foram utilizados 20 alunos de Psicologia, com dois grupos experimentais, que foram submetidos ao treino de leitura compreensiva e leitura criativa. Os dados obtidos demonstraram a eficiência do treino em ambos os grupos. Quanto à generalização do treino, pode-se dizer que o grupo que passou pelo treino de compreensão apresentou melhor desempenho em criticidade textual do que o grupo submetido ao treino de criticidade. Pode-se concluir, ainda, que ambos os grupos produziram a melhora dos comportamentos de leitura criativa, leitura compreensiva e motivação de leitura.

Neste estudo, usa-se a posição teóricometodológica cognitiva comportamental, já que a literatura de área, na sua maioria, está dentro do enfoque cognitivista.

Assim, pretende-se oferecer uma instrução coletiva em criticidade textual para os alunos, e, dentro da posição teórico-metodológico assumida, há uma classe de respostas na presença de estímulos discriminativos que têm determinadas conseqüências no ambiente, e que, planejadas, devem fortalecer a classe de respostas de destacar aspectos importantes. Dessa forma, as condições antecedentes usadas foram o uso de questões como estímulos discriminativos para o leitor responder e utilizar a audiência (reforçamento social) fornecida pela experimentadora para o desenvolvimento da criticidade e da compreensão textual (Staats, 1968).

Neste trabalho, o conceito de criticidade será considerado dentro do contexto teóricometodológico assumido, e, portanto, será focalizado como uma forma de resposta dos alunos ao controle exercido pela autoridade do texto. Assim, também neste estudo, será definida a leitura crítica como a habilidade do estudante universitário em verificar se as informações contidas no texto estão baseadas em fatos ou em opiniões do autor, mantendo o nível de adequação ao contexto da leitura. 
Apesar de as habilidades mais complexas de leitura serem enfatizadas pelos educadores, pode-se concluir que são escassas as pesquisas com universitários nessa área. Pode-se dizer também que são necessários estudos que possam dar algum esclarecimento quanto ao treino dessas habilidades em sala de aula e que possam explicitar melhor o efeito do uso de questões e da ciência do desempenho sobre esses comportamentos nos universitários.

Dentro do referencial até aqui apresentado, foram formulados como objetivos para a presente pesquisa :

1) avaliação de compreensão de textos comparando cinco grupos de estudantes de pós-graduação lato-sensu;

2) teste da eficiência de um treino de criticidade textual comparando os cinco grupos.

\section{Método}

Desse modo, foi construído o método conforme a posição teórico-metodológica cognitiva comportamental, que se encontra descrita abaixo.

\section{Participantes}

O número de participantes deste trabalho foi de 20 alunos de duas classes que freqüentavam um curso de Metodologia do Ensino Superior, ministrado pela mesma professora aos alunos de pós-graduação lato sensu de uma universidade municipal do
Estado de São Paulo. Os grupos foram assim constituídos: G.I, com 4 alunos de humanas, média de idade, 44 anos; G.II, com igual número de estudantes de Economia, com média de idade de 38 anos; G.III, da área de Engenharia, idade de 31 anos em média; G.IV, da saúde, com idade média de 34 anos, e o G.V, da área de Letras e idade média de 32 anos.

O número de participantes nos diversos grupos é reduzido pelo fato de os alunos faltarem muito aos cursos. Foi considerado como critério a presença de $100 \%$ no Curso de Treino de Leitura.

\section{Material}

Como medida de compreensão de leitura, foi usada a técnica de Cloze, criada por Taylor (1953). Foram estabelecidas 40 lacunas, seguindo-se o modelo-padrão. A primeira e última oração não sofreram apagamentos. Foram utilizados dois trechos desconhecidos pelos alunos do livro de Bomtempo, Hussein e Zamberlan, de 1986. Essa técnica foi usada em nosso meio por Santos (1989) e nos vários estudos dos livros organizados por Witter (1997, 1999, 2004). Os textos comuns de leitura foram usados no treino e enfocavam a aprendizagem verbal; outro critério foi que os textos tivessem gradativamente aumentados o número de páginas.

Quase todas as folhas de respostas usadas no treino variaram de uma até quatro questões semelhantes às usadas no Teste de Criticidade, que consta do estudo de Hussein (2007). As questões usadas no treino foram as seguintes: 1) O autor define o problema justificando-se 
com fatos ou baseia-se em opiniões? Por quê?; 2) A temática afirmada pelo autor quanto às implicações educacionais está baseada em fatos ou em opiniões? Por quê?; 3) A argumentação do autor quanto às implicações teóricas está justificada em fatos ou opiniões? Por quê? e 4) As premissas e conclusões do problema estão baseados em fatos ou em opiniões? Por quê?

\section{Procedimento}

Foram aplicados em cada aula no pré e pósteste para os cinco grupos o teste Cloze.

O procedimento constou de cinco sessões de treino. A duração de cada sessão foi de aproximadamente uma hora aula. O planejamento foi feito de modo a viabilizar a concretização da coleta de dados de pesquisa dentro do espaço de tempo disponível em um semestre letivo.

Todos os grupos receberam o treino de criticidade. O treino iniciou-se simultaneamente para todos os grupos, e foi constituído de duas fases: Fase 1 e Fase 2.

A Fase 1 ( 1 aula) do treino de criticidade constou da leitura individual de um texto programado: Questões: Importância distinção entre fato e opinião (Cheves, 1973), tendo como objetivos levar os alunos a indicarem a relevância das questões e a distinguir o que é fato e o que é opinião no material lido. Considerou-se a necessidade de um texto preliminar na medida em que se constatou, no estudo de Hussein (1999, 2007), que os estudantes apresentaram um grande número de respostas inadequadas durante e após o treino de criticidade textual, apesar do progresso em relação ao pré, indicando possivelmente a necessidade de prérequisitos. Também isso justifica a inclusão, na Fase 2 ( 4 aulas), da folha de instruções para o treino de criticidade que o aluno universitário sempre recebia para ler antes de realizar cada sessão de treino de leitura crítica e era mantida durante toda a sessão. Essa folha de instruções começa por considerações sobre a importância da atitude de curiosidade e de crítica. Depois ela apresenta a definição de fato científico em Psicologia, seguido de vários exemplos extraídos de diversos textos. Em segundo lugar, a folha define o que é opinião e mostra vários exemplos de autores que indicam esse comportamento. Essa instrução ainda conceitua inferência, seguido de alguns trechos para exemplificar a definição. Finalmente, ela apresenta, no final, a síntese desses três conceitos e indica para o leitor que o treino de leitura crítica proposto tem por objetivo desenvolver a habilidade de distinguir fatos de opiniões.

Na fase 2 ( 4 aulas), foram apresentadas todas as quatro questões após a leitura do texto. Após a obtenção das respostas, a aplicadora recolhia o material e agradecia o trabalho do aluno.

Depois de cada treino, a experimentadora corrigia as respostas e as devolvia para os alunos conhecerem a avaliação de seu desempenho, antes de passarem para nova sessão de treino. Era também uma forma de reforçamento dado pela pesquisadora na forma escrita, atuando a mesma como uma audiência privilegiada.

\section{Resultados}

Em relação ao Teste Cloze, foi considerada como resposta certa o vocábulo que correspondesse exatamente ao que havia sido omitido no texto. 
Os dados foram submetidos ao Teste de Wilcoxon e Wilcox (1964) para a comparação intergrupos e ao Teste de Wilcoxon para comparação intra-grupos, usando-se o nível de significância de 0,05 .

O primeiro teste é não paramétrico, e é indicado para a comparação de vários grupos de tratamento e para quando não se dispõe de informação sobre a curva de distribuição, mas podese contar com igual número de observações para cada grupo de tratamento.

Foi proposta como hipótese nula a igualdade entre os grupos (Ho:G.I=G.II=G.III=G.IV=G.V), e como hipótese alternativa que os grupos comparados difeririam entre si $(\mathrm{H} 1: \mathrm{G} . \mathrm{I} \neq \mathrm{G}$.II $\neq \mathrm{G}$. III $\neq \mathrm{G}$. $I V \neq G . V)$, porém sem especificar a direção dessa diferença, o que constituía, portanto, um teste bicaudal. Dado que $n=4$ e $K=5$, o valor da diferença crítica, nesse caso, é de 45,6.

Os resultados encontram-se descritos em relação a cada teste usado no presente estudo e estão expressos nas Tabelas 1 e 2 .

Tabela 1. Comparação Inter grupos no Teste Cloze (acerto, erro, em branco ) no pré-teste $(n=4$; $K=5$ e d.c $=45,6)$.

\begin{tabular}{c|ccccc}
\multicolumn{7}{c}{ Acerto } \\
\hline \multicolumn{7}{c}{ G.III } & G.IV & G.I & G.V & G.II \\
G.III & 59,5 & 43,0 & 40,5 & 36,5 & 30,0 \\
\cline { 2 - 6 } 59,5 & - & - & - & - & - \\
G.IV & 16,5 & - & - & - & - \\
43,0 & & & & & \\
G.I & 19,0 & 2,5 & - & - & - \\
40,5 & & & & & \\
G.V & 23,0 & 16,5 & 4,0 & - & - \\
36,5 & & & & & \\
G.II & 29,5 & 10,5 & 10,5 & 6,5 & - \\
30,0 & & & & & \\
\hline
\end{tabular}

\begin{tabular}{cccccc}
\multicolumn{7}{c}{ Erro } \\
\hline \multicolumn{7}{c}{ G.II } & G.V & G.I & G.I & G.III \\
G.II & 69,0 & 44,0 & 36,5 & 36,5 & 27,0 \\
\cline { 2 - 7 } 69,0 & - & - & - & - & - \\
G.V & 25,0 & & & & \\
44,0 & & - & - & - & - \\
G.I & 32,5 & 7,5 & - & - & - \\
36,5 & & & & & \\
G.I & 35,5 & 10,5 & 3,0 & - & - \\
36,5 & & & & & \\
G.III & 42,0 & 17,0 & 9,5 & 6,5 & - \\
27,0 & & & & & \\
\hline
\end{tabular}

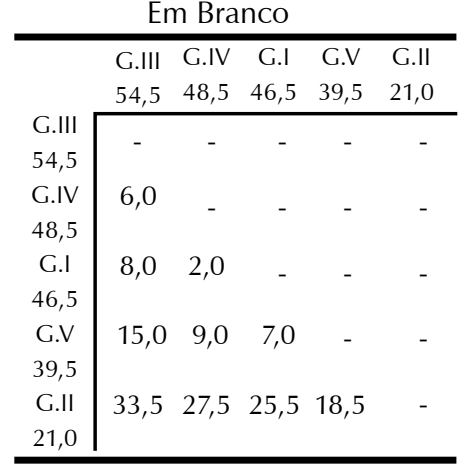

Tabela 2. Comparação Inter grupos no Teste Cloze (acerto, erro, em branco ) no pós-Teste $(n=4$; $K=5$ e d.c $=45,6)$.

\begin{tabular}{cccccc}
\multicolumn{7}{c}{ Acerto } \\
\hline \multicolumn{7}{c}{ G.III } & G.IV & G.V & G.I & G.II \\
G.III & 51,5 & 49,5 & 44,0 & 38,5 & 26,5 \\
\cline { 2 - 6 } 51,5 & - & - & - & - & - \\
G.IV & 2,0 & & & & \\
49,5 & & - & - & - & - \\
G.V & 7,5 & 5,5 & - & - & - \\
44,0 & & & & & \\
G.I & 13,0 & 11,0 & 5,5 & - & - \\
38,5 & & & & & \\
G.II & 25,0 & 23,0 & 17,5 & 12,0 & - \\
26,5 & & & & & \\
\hline
\end{tabular}

\begin{tabular}{rrrrrr}
\multicolumn{7}{c}{ Erro } \\
\hline \multicolumn{7}{c}{ G.II } & G.I & G.III & G.V & G.IV \\
G.II & 49,0 & 47,0 & 47,0 & 42,5 & 24,5 \\
\cline { 2 - 6 } 49,0 & - & - & - & - & - \\
G.I & 2,0 & & & & \\
47,0 & & - & - & - & - \\
G.III & 2,0 & & - & - & - \\
47,0 & & & & & \\
G.V & 6,5 & 4,5 & 4,5 & - & - \\
42,5 & & & & & \\
G.IV & 24,5 & 22,5 & 22,5 & 18,0 & - \\
24,5 & & & & & \\
\hline
\end{tabular}

\begin{tabular}{cccccc}
\multicolumn{7}{c}{ Em Branco } \\
\hline \multicolumn{7}{c}{ G.IV } & G.II & G.I & G.V & G.III \\
G.IV & 56,5 & 51,0 & 48,0 & 33,5 & 21,0 \\
\cline { 2 - 6 } 56,5 & - & - & - & - & - \\
G.II & 5,5 & & & & \\
51,0 & & - & - & - & - \\
G.I & 8,5 & 3,0 & & & \\
48,0 & & & - & - & - \\
G.V & 23,0 & 17,5 & 14,5 & - & - \\
33,5 & & & & & \\
G.III & 35,5 & 30,0 & 27,0 & 12,5 & - \\
21,0 & & & & & \\
\hline
\end{tabular}


Conforme os dados das Tabelas 1 e 2 indicam, as comparações das emissões de respostas quanto ao número de acertos, erros e respostas em branco do Teste Cloze no pré-teste e no pós-teste não evidenciaram diferenças entre os grupos estudados.

Tabela 3. Descrição dos níveis de leitura no pré e pós em todos os grupos.

\begin{tabular}{|c|c|c|c|c|c|c|}
\hline \multirow{2}{*}{$\begin{array}{l}\text { Classificação } \\
\text { Grupos / Fases }\end{array}$} & \multicolumn{2}{|c|}{$\begin{aligned} & \text { Bom } \\
+ & \text { de } 57 \%\end{aligned}$} & \multicolumn{2}{|c|}{$\begin{array}{c}\text { Regular } \\
\text { De } 44 \text { de } 57 \% \\
\end{array}$} & \multicolumn{2}{|c|}{$\begin{array}{c}\text { Fraco } \\
\text { Menos de } 44 \%\end{array}$} \\
\hline & Pré & Pós & Pré & Pós & Pré & Pós \\
\hline $\begin{array}{l}\text { G. I } \\
\text { Humanas } \\
\text { G.II }\end{array}$ & $100 \%$ & $100 \%$ & - & - & & - \\
\hline $\begin{array}{l}\text { Economia } \\
\text { G.III }\end{array}$ & $100 \%$ & $50 \%$ & - & $50 \%$ & & - \\
\hline $\begin{array}{l}\text { Engenharia } \\
\text { G.IV }\end{array}$ & $75 \%$ & $50 \%$ & $25 \%$ & $25 \%$ & & $25 \%$ \\
\hline $\begin{array}{l}\text { Saúde } \\
\text { G.V }\end{array}$ & $100 \%$ & $50 \%$ & - & $25 \%$ & & $25 \%$ \\
\hline Letras & $75 \%$ & $75 \%$ & $25 \%$ & - & & $25 \%$ \\
\hline
\end{tabular}

Santos (1989) afirma que uma vantagem da técnica de Cloze no diagnóstico da compreensão em leitura é destacada por Cunningham e Cunningham (1978) ao concluirem que os resultados percentuais alcançados por um estudante, em um determinado trecho, podem dar ao professor o nível de sua compreensão com relação ao texto todo. Assim, na classificação proposta por Bormuth (1968), o aluno que conseguir um acerto de mais de 57\% é capaz de ler o texto integral de forma independente (bom). Aqueles alunos que acertarem de 44 a 57\% das respostas serão capazes de ler se uma adequada orientação do professor acompanhar a leitura (regular), e aqueles cujos percentuais de acerto não sejam suficientes para alcançar 44\%, provavelmente não conseguirão ler o texto, a não ser com muita ajuda do professor (fraco).

A Tabela 3 descreve o nível de leitura no pré e pós-teste em relação a cada grupo. Constatou-se que o desempenho é quase homogêneo, o que, portanto, indica que são bons leitores tanto no pré como no pós. Eles conseguiram quase sempre um acerto de mais de $57 \%$ o que revela que são capazes de ler o texto integral e de forma independente.

A comparação intra-grupos teve por objetivo verificar o efeito do procedimento de treino de criticidade textual sobre o desempenho dos alunos universitários no teste usado. Esse é um teste não paramétrico, sendo indicada a sua aplicação para o caso de amostras dependentes entre si, para as quais não se dispõe de dados suficientes para pressupor uma distribuição normal e não se leve em consideração a média, mas sim, o desempenho de cada participante no grupo.

Levantou-se, por hipótese de nulidade, que pré = pós e, por hipótese alternativa, estabeleceuse que, como os participantes foram submetidos a algum procedimento de treino, teriam um desempenho superior no pós-teste quando comparado ao pré, ou seja, há : pré < pós, sendo estabelecida uma base unidirecional. 
Realizaram-se comparações entre o pré e o pós para verificar a eficiência do treino recebido pelos alunos, que se encontram expressas na Tabela 4.

Tabela 4. Comparação Intra-grupos no Teste de Cloze (acerto, erro e em branco) no pré e pós (nível de significância de 0,05).

\begin{tabular}{lcccc}
\hline \multirow{2}{*}{ Instrumentos } & Comparação & \multicolumn{4}{l}{ Pré x Pós } \\
\cline { 2 - 5 } & $\mathrm{N}$ & TC & TO & $\begin{array}{l}\mathrm{H} 0=\text { Pré }=\text { Pós } \\
\mathrm{H} 1=\text { Pré }<\text { Pós }\end{array}$ \\
\hline Cloze - acerto & 19 & 92 & 20,5 & Rejeitada \\
Cloze - erro & 20 & 104 & -66 & Rejeitada \\
Cloze - em branco & 16 & 60 & -47 & Rejeitada \\
\hline
\end{tabular}

Como o $\mathrm{N}$ de cada grupo de tratamento foi 4, e o Teste de Wilcoxon requer o mínimo para o tratamento de 6 , resolveu-se aglutinar os dados dos participantes de todos os grupos para se obter um $\mathrm{N}$ maior, que foi de 20 alunos.

Assim, de acordo com os dados da Tabela 4, verificou-se, no Teste Cloze, que a hipótese nula foi rejeitada para as três categorias de respostas (acerto, erro e em branco). Verificou-se diferença entre a fase antes do treino e a fase posterior, o que já era esperado. Em relação a acertos, o progresso foi significativo $(T o=20,5)$, tendo havido, em relação aos erros, uma redução substancial $(\mathrm{To}=-66)$; também ocorreram no pós menos respostas em branco $(\mathrm{To}=-47)$. Vale dizer que o treino foi eficiente igualmente para todas as áreas de conhecimento pesquisadas.

\section{Discussão}

Em primeiro lugar, atendeu-se à ordem de apresentação dos objetivos. O primeiro objetivo verifica a avaliação de compreensão de textos comparando cinco grupos de estudantes de pósgraduação lato-sensu. A seguir, foi verificada a eficiência de treino de criticidade textual desses estudantes.

Os dados referentes às comparações intergrupos foram considerados primeiramente, e, depois, as comparações intragrupos. Assim, para se verificar o atendimento dos objetivos, focalizaram-se as comparações entre o pré e o pós-teste.

Focalizando a comparação intergrupos, os dados mostraram que não houve diferenças significativas entre os grupos quanto à produção de compreensão textual, e os resultados intragrupos revelaram que os alunos indicaram melhoria desse comportamento em todas as medidas de compreensão. 
Outro aspecto sugerido para o ensino de criticidade textual e criatividade textual é a utilização de um método que possibilite a participação para uma audiência reforçadora constituída pela pesquisadora ao analisar o trabalho realizado pelo leitor, assim como que o conduzia a contrastar e a comparar diferentes pontos de vista (Okibayashi, 2004; Richards, 2006).
Esses dados apóiam os resultados obtidos por Boodt (1984) com escolares, que evidenciaram que as habilidades de compreensão textual parecem ser desenvolvidas com o ensino de leitura crítica. Também os dados do presente trabalho apóiam os resultados encontrados por Hussein (2008) com escolares da 5a série, em que se constatou que o desenvolvimento da compreensão parece ter sido independente da variável experimental utilizada, no caso, a instrução recebida (leitura crítica, leitura criativa e reprodução de textos). Desse modo, os resultados sugerem que essa variável foi relevante em termos de controlar a produção desse comportamento. Outro estudo de Hussein (2007) que corrobora os resultados do presente estudo com alunos universitários de Psicologia evidenciou que a compreensão textual foi independente do treino de compreensão e de criticidade textual recebido. Parece que o interesse e o nível de compreensão foram atendidos no presente estudo, e o material de leitura pode ter sido uma variável importante.

Os dados do presente trabalho parecem demonstrar a eficiência do treino de criticidade textual para o desenvolvimento da compreensão textual nos sujeitos universitários a despeito do número reduzido de sessões; portanto, tudo recomenda o procedimento usado.

Este estudo parece comprovar o uso do modelo de uso de questões como forma de conduzir o treino de compreensão textual, demonstrando a importância da sua utilização para a emissão de respostas críticas seguidas de reforçamento social liberado pela audiência no desenvolvimento e na manutenção da compreensão de leitura.
Assim, esses resultados parecem comprovar os obtidos por Hussein (1999, 2007), que usou programas que incluem questões que requerem processos cognitivos superiores para o ensino de compreensão textual de alunos universitários.

Aprender a perguntar usando questões cheias de significado é um componente-chave do pensamento crítico em face de textos (Wade, 1995). A qualidade das questões feitas à classe determina o nível de pensamento que ocorre (King, 1995). Assim, pesquisas sobre o ensino de Psicologia enfatizam o ensino de habilidades de questionamento aos alunos universitários para desenvolver habilidades mais complexas de leitura.

Outro aspecto sugerido para o ensino de criticidade textual e criatividade textual é a utilização de um método que possibilite a participação para uma audiência reforçadora constituída pela pesquisadora ao analisar o trabalho realizado pelo leitor, assim como que o conduzia a contrastar e a comparar diferentes pontos de vista (Okibayashi, 2004; Richards, 2006).

Logo, o procedimento utilizado neste estudo é recomendado para alunos de pós-graduação em Psicologia como iniciação científica. Seria interessante compará-lo com outros estudos que usassem questões sobre metodologia científica em cursos de graduação e de pósgraduação em Psicologia como os citados por Harlpern e Nummedal (1995) em seu estudo de revisão de leitura crítica.

Dessa forma, também seria importante realizar pesquisas que demonstrassem o efeito 
do método remediativo da compreensão sobre a generalização para outros comportamentos bem como para a situação acadêmica.

Pode-se concluir, portanto, pela necessidade de estudos de réplica e também pela necessidade de estudos posteriores sobre a influência da compreensão sobre o desenvolvimento de leitura crítica e vice-versa em estudantes universitários com critérios mais precisos em relação à motivação e ao nível de compreensão.

Carmen Lúcia Hussein

Universidade de Taubaté

Doutora em Psicologia do Escolar pelo IPUSP

\section{Endereço para envio de correspondência:}

Av. Dr. Francisco de Paula Vicente de Azevedo, no 1439 - Pq Continental - São Paulo-SP - Brasil CEP 05325-180

E-mail-carmenhussein@terra.com.br 
Applegate, M. D., Quinn, K. B., \& Applegate, A. J. (2002). Levels of thinking required by comprehension questions in informal reading inventories. The Reading Teacher, $56(2), 18-22$.

Bomtempo, E., Hussein, C. L., \& Zamberlan, M. A. T. (1986). Psicologia do brinquedo: aspectos teóricos e metodológicos. São Paulo: Edusp.

Boodt, G. M. (1984). Critical listeners become critical readers in remedial reading class. The Reading Teacher, 37(4), 390-394.

Bormuth, J. R. (1968). Close test readability: Criterion reference scores. Journal of Educational Measuring, 5, 189-196.

Cheves, D. A. P. (1973). An experiment in developing the ability to make inferences and to ask questions which require inference in sixt grade students. Kansas City, MO: University of Missouri.

Connor-Greene, P. A. (2005). Fostering meaningful classroom discussion: Student-generated questions, quotations and talking points. Teaching of Psychology, 32(3), 173-175

Cunningham, J. W., \& Cunningham, P. M. (1978). Validating a limited Cloze procedure. Journal of Reading Behavior, $1(2), 211-214$

Douglas, N. L. (2000). Enemies of critical thinking: Lessons from social psychology research. Reading Psychology, 21,129-141.

Ferreira, S. P. A., \& Dias, M. J. B. B. (2002). A escola e o ensino da leitura. Psicologia em Estudo, 7(1), 39-49.

Halpern, \& Nummedal, P. (1995). Psychologists teach critical thinking. Teaching of Psychology, 22(1), 3-98.

Hussein, C. L. (1999). Teste de procedimentos de treino e generalização de leitura crítica e criativa: um estudo experimental com universitários. Estudos de Psicologia (Campinas), 16(2), 16-27.

Hussein, C. L. (2007). Avaliação de modalidades de treino de leitura compreensiva e leitura crítica: um estudo com universitários. Trabalho submetido à publicação.

Hussein, C. L. (2008). Leitura crítica e criativa: ensino e aprendizagem. Rio de Janeiro: C.B.J.E.

King, A. (1995). Inquiring really do want to know: Using questioning to teach critical reading. Teacher of Psychology, 22(1), 1316

Marini, A. (1986). Compreensão de leitura no ensino superior: teste de um programa para treino de habilidades. Tese de Doutorado, Instituto de Psicologia, Universidade de São Paulo, São Paulo.

Okibayashi, Y. (2004). Students'critical reading of articles: Effects of guidance and group discussion. Japanese Journal of Educational Psychology, 52(3), 242-254.

Oliveira, K. L., \& Santos, A. A. A. (2005). Compreensão em leitura e avaliação da aprendizagem em universitários. Psicologia: Reflexão e Crítica, 18(1), 118-124.
Oliveira, K. L., \& Santos, A. A. A. (2006). Compreensão de textos e desempenho acadêmico. Psic-Revista de Psicologia da Vetor Editora, 7(1), 19-27.

Pailliotet, A. W., Semali, L., Rodenberg, R. K., Giles, J. K., \& Macaul, S. L. (2000). Intermediality: Bridge to critical media literacy. The Reading Teacher, 54(2), 2018-2019.

Richards, J. C. (2006). Question, connect, transform (QCT)I: A strategy to help middle school students engage critically with historical fiction. Reading and Writing Quarterly: Overcoming Learning Difficulties, 22(2), 193-198.

Sainsbury, M. (2006). Review of learning to read crittically in language and literacy. Educational Research, 48(3), 334335.

Sampaio, I. S., \& Santos, A. A. A. (2002). Leitura e redação entre universitários: avaliação de um programa de intervenção. Psicologia em Estudo, 7(1), 31-38.

Santos, A. A. A. (1989). Leitura entre universitários: diagnóstico e remediação. Tese de Doutorado, Instituto de Psicologia, Universidade de São Paulo, São Paulo.

Santos, A. A. A. (1991). Desempenho em leitura: um estudo diagnóstico da compreensão e hábitos de leitura entre universitários. Estudos de Psicologia (Campinas), 8(1), 6-19.

Santos, A. A. A., Primi, R., Taxa, F., Vendramini, C. M. M. (2002). O Teste de Cloze na avaliação da compreensão em leitura. Psicologia: Reflexão e Crítica, 15(3), 549-560.

Staats, A. W. (1968). Learning, language and cognition. New York: Holt, Rinehart and Winston.

Taylor, W. L. (1953). Cloze procedure: A new tool for measuring readability. Journalism Quarterly, 30, 415-433.

Tessaro, N. S. (2004). Leitura na vida de universitários: estudo comparativo entre instituições de ensino superior. In G. P. Witter (Ed.), Leitura e Psicologia (pp. 45-76). Campinas, SP: Alínea.

Wade, C. (1995). Using writing to develop and assess critical thinking. Teaching of Psychology, 22(1), 24-28.

Wilcoxon, F., \& Wilcox, R. A. (1964). Some rapide approximate statistical procedures. New York: Pub Lederle Laboratories.

Witter, G. P. (1997). Leitura e universidade. In G. P. Witter (Ed.), Psicologia - leitura e universidade (pp. 9-18). Campinas, SP: Alínea.

Witter, G. P. (1999). Leitura: textos e pesquisas. Campinas, SP: Alínea.

Witter, G. P. (2004). Leitura e psicologia. Campinas, SP: Alínea.

Wolf, M. K., Crosson, A. C., \& Resnick, L. B. (2005). Classroom talk for rigorous reading comprehension instruction. Reading Psychology, 26, 27-53. 